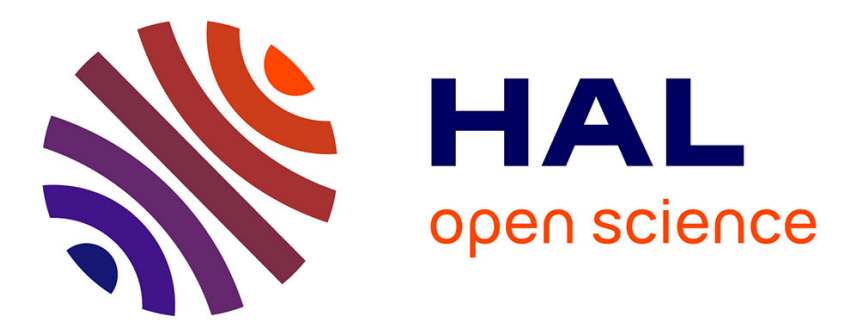

\title{
Conservation Laws with Unilateral Constraints in Traffic Modeling
}

\author{
Rinaldo M. Colombo, Paola Goatin, Massimiliano D Rosini
}

\section{To cite this version:}

Rinaldo M. Colombo, Paola Goatin, Massimiliano D Rosini. Conservation Laws with Unilateral Constraints in Traffic Modeling. De Bernardis E. and Spigler R. and Valente V. Applied and Industrial Mathematics in Italy III, 82, Series on Advances in Mathematics for Applied Sciences, pp.244-255, 2009, 10.1685/CSC09XXX . hal-00765427

\section{HAL Id: hal-00765427 https://hal.inria.fr/hal-00765427}

Submitted on 14 Dec 2012

HAL is a multi-disciplinary open access archive for the deposit and dissemination of scientific research documents, whether they are published or not. The documents may come from teaching and research institutions in France or abroad, or from public or private research centers.
L'archive ouverte pluridisciplinaire HAL, est destinée au dépôt et à la diffusion de documents scientifiques de niveau recherche, publiés ou non, émanant des établissements d'enseignement et de recherche français ou étrangers, des laboratoires publics ou privés. 


\title{
Conservation Laws with Unilateral Constraints in Traffic Modeling
}

\author{
Rinaldo M. Colombo ${ }^{1}$, Paola Goatin ${ }^{2}$, Massimiliano D. Rosini ${ }^{3}$ \\ ${ }^{1}$ Dipartimento di Matematica \\ Via Branze 28, I-25123 Brescia, Italy \\ rinaldo@ing. unibs.it \\ ${ }^{2}$ I.S.I.T.V. \\ B.P. 56, 83162 La Valette du Var Cedex, France \\ goatin@univ-tln.fr \\ ${ }^{3}$ IM PAN \\ ul. Sniadeckich 8, 00956 Warszawa, Poland \\ mrosini@univaq. it
}

Keywords: Traffic Modeling, Conservation Laws, Nonclassical Shocks

\begin{abstract}
Macroscopic models for both vehicular and pedestrian traffic are based on conservation laws. The mathematical description of toll gates along roads or of the escape dynamics for crowds needs the introduction of unilateral constraints on the observable flow. This note presents a rigorous approach to these constraints, and numerical integrations of the resulting models are included to show their practical usability.
\end{abstract}

\section{Introduction}

The classical Lighthill-Whitham [22] and Richards [23] traffic model is based on the following simple assumptions:

1. The total number of vehicles is conserved.

2. The speed at a point is a function of the density at that point.

Both assumptions appear perfectly suitable also for the macroscopic modeling of crowds. From the analytical point of view, in 1D, these assumptions lead to the conservation law

$$
\partial_{t} \rho+\partial_{x}[\rho v(\rho)]=0
$$

where the speed law $v=v(\rho)$ plays a role analogous to that played by the equation of state for Euler equations of gas dynamics. 
Along a highway, a toll gate acts as a unilateral constraint on the flow. Indeed, call $q$ the given maximal flow of vehicles that can pass through the gate sited at, say, $x=0$. Then, the resulting traffic can be reasonably described through (1.1) supplemented with

$$
\rho(t, 0) v(\rho(t, 0)) \leq q .
$$

(All BV functions, such as solutions to conservation laws, are assumed to be continuous from the left, so that their value is well defined at any point).

Similarly, consider a crowd escaping along a corridor through an exit. Let $q$ denote the maximal through flow at the exit sited, say, at $x=0$. Then, again, equation (1.1) supplemented with (1.2) provides a simple though effective description of the escape dynamics. For other related descriptions of pedestrian dynamics, see $[14,15,16,17,18,19]$.

Remark, however, that there is a key qualitative difference in the real evolutions of the two preceding examples. On one hand, the efficiency of the toll gate is not affected by the amount of vehicles lining up. On the other hand, as is well known, the crowd heading towards the exit may cause overcompression phenomena, possibly leading to panic, that may well reduce the efficiency of the exit. Therefore, in the case of pedestrians, we let the evolution of (1.1) be governed by nonclassical shocks.

The next section is devoted to the analytical results on (1.1)-(1.2). Section 3 deals with the toll gate model while Section 4 is devoted to the case of pedestrians.

\section{Analytical Results}

Following [8], we consider the Constrained Cauchy Problem (CCP)

$$
\left\{\begin{array}{l}
\partial_{t} \rho+\partial_{x} f(\rho)=0 \\
\rho(0, x)=\rho_{o}(x) \\
f(\rho(t, 0)) \leq q(t)
\end{array}\right.
$$

under the standard assumptions

$(\mathrm{CCP} 1) f \in \mathbf{C}^{\mathbf{0}, \mathbf{1}}([0, R] ; \mathbb{R}), f(0)=f(R)=0$, there exists a $\left.\bar{\rho} \in\right] 0, R[$ such that $f^{\prime}(\rho)(\bar{\rho}-\rho)>0$ for a.e. $\rho \in[0, R]$.

$(\mathrm{CCP} 2) q \in \mathbf{B V}\left(\mathbb{R}^{+} ;[0, f(\bar{\rho})]\right)$.

Note that in the case $f(\rho)=\rho v(\rho),(2.3)$ reduces to the Cauchy Problem for (1.1)-(1.2).

To state the next well posedness result, it is useful to introduce the translation $\mathcal{T}_{t}$ through $\left(\mathcal{T}_{t} q\right)(\tau)=q(\tau+t)$. Below we define a map $\mathcal{S}: \mathbb{R}^{+} \times$ 
$\mathcal{D} \mapsto \mathcal{D}, \mathcal{D}$ being a suitable subset of $\mathbf{L}^{\mathbf{1}}$ containing the initial data of (2.3). We then denote by $\overline{\mathcal{S}}$ the map $\overline{\mathcal{S}}: \mathbb{R}^{+} \times \overline{\mathcal{D}} \mapsto \overline{\mathcal{D}}$ defined by $\overline{\mathcal{S}}_{t}(\rho, q)=$ $\left(\mathcal{S}_{t} \rho, \mathcal{T}_{t} q\right)$ with $\overline{\mathcal{D}}=\mathcal{D} \times \mathbf{B V}$.

Following $[7,8,25]$, we use the nonlinear mapping

$$
\Psi(\rho)=\operatorname{sign}(\rho-\bar{\rho})(f(\bar{\rho})-f(\rho)) .
$$

Recall that by Riemann Problem we mean the following particular Cauchy Problem, see also [3, Chapter 5]:

$$
\left\{\begin{array}{l}
\partial_{t} \rho+\partial_{x} f(\rho)=0 \\
\rho(0, x)= \begin{cases}\rho_{l} & \text { if } x<0 \\
\rho_{r} & \text { if } x>0\end{cases}
\end{array}\right.
$$

Theorem 2.1. [8, Theorem 3.4] Let (CCP1) and (CCP2) hold. Then, for every constraint $q \in \mathbf{B V}\left(\mathbb{R}^{+} ;[0, f(\bar{\rho})]\right)$ there exists a map $\mathcal{S}: \mathbb{R}^{+} \times \mathcal{D} \mapsto \mathcal{D}$ such that

$(S 1) \mathcal{D} \supseteq\left\{\rho \in \mathbf{L}^{\mathbf{1}}(\mathbb{R} ;[0, R]): \Psi(\rho) \in \mathbf{B V}(\mathbb{R} ; \mathbb{R})\right\}$;

(S2) $\overline{\mathcal{S}}$ is a Semigroup, i.e. $\overline{\mathcal{S}}_{0}=\operatorname{Id}$ and $\overline{\mathcal{S}}_{t_{1}} \circ \overline{\mathcal{S}}_{t_{2}}=\overline{\mathcal{S}}_{t_{1}+t_{2}}$;

(S3) $\mathcal{S}$ is non expansive in $\rho$, i.e. for all $\rho_{1}, \rho_{2} \in \mathcal{D}$

$$
\left\|\mathcal{S}_{t} \rho_{1}-\mathcal{S}_{t} \rho_{2}\right\|_{\mathbf{L}^{1}} \leq\left\|\rho_{1}-\rho_{2}\right\|_{\mathbf{L}^{1}}
$$

(S4) if $\rho_{o}$ and $q$ are piecewise constant, then for $t$ sufficiently small, $\mathcal{S}_{t} \rho_{o}$ coincides with the gluing of the solutions to standard Riemann problems centered at the points of jump of $\rho_{o}$ and to (2.3) at $x=0$;

(S5) for all $\rho_{o} \in \mathcal{D}$, the orbit $t \mapsto \mathcal{S}_{t} \rho_{o}$ yields a weak entropy solution to (2.3), according to [8, definitions 3.1 and 3.2].

The proof uses the standard technique of wave front tracking, see [3, Chapters 6 and 7], and is deferred to [8]. The above statements (S1)-(S4) are clearly modeled on the definition of Standard Riemann Semigroup, see [3, Definition 9.1] and provide an analogue to it in the present constrained (and non autonomous) setting. The Lipschitz estimate (S3) is proved with suitable modifications of the classical techniques in [20] or [3, Section 6.3].

It is now easy to tackle the Initial Boundary Value Problem

$$
\left\{\begin{array}{lrl}
\partial_{t} \rho+\partial_{x} f(\rho)=0 & (t, x) & \in \mathbb{R}^{+} \times \mathbb{R}^{-} \\
\rho(0, x)=\rho_{o}(x) & x & \in \mathbb{R}^{-} \\
f(\rho(t, 0)) \leq q(t) & t & \in \mathbb{R}^{+}
\end{array}\right.
$$


Indeed, as in the case of the Riemann Problem, a solution to (2.5) is obtained restricting to $x<0$ a solution to (2.3) with initial data, say, $\rho_{o}(x)=0$ for $x>0$. The extension to the general Initial Boundary Value Problem

$$
\left\{\begin{array}{lrl}
\partial_{t} \rho+\partial_{x} f(\rho)=0 & (t, x) & \in \mathbb{R}^{+} \times[0, L] \\
\rho(0, x)=\rho_{o}(x) & x & \in[0, L] \\
f(\rho(t, 0))=f_{o}(t) & t & \in \mathbb{R}^{+} \\
f(\rho(t, L)) \leq q(t) & t & \in \mathbb{R}^{+}
\end{array}\right.
$$

is immediate, see $[9,13]$ and the references therein as a general reference on Initial Boundary Value Problems for scalar conservation laws.

In the case of pedestrians, the model presented in [10], see also [11,24], is based on a flow like that in Figure 1. Note that this particular shape of the
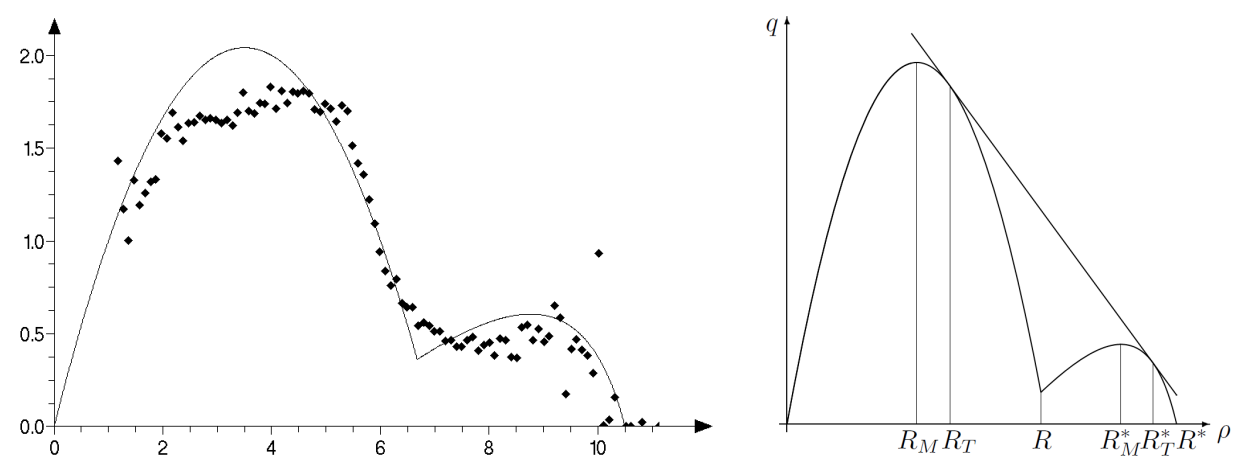

Fig. 1. Left, a flow satisfying the requirements of Theorem 2.2. Superimposed are the experimental measurements from [16]. Crowd density, $\rho$, is on the horizontal axis and flow, $\rho v$, on the vertical one. Right, another possible flow and the notation.

fundamental diagram was first postulated in [10] and then experimentally verified in [16]. Moreover, the evolution of the solutions to (1.1) is governed through the introduction of nonclassical shocks. That is, a Riemann Solver $\mathcal{R}$ is defined, which assigns to every pair $\rho_{l}, \rho_{r}$ the self similar weak solution to the Riemann Problem (2.4) computed at time, say, $t=1$.

As it is usual when dealing with nonclassical scalar conservation laws, see [21, Chapter II], we need first to introduce the auxiliary function $\psi$, see Figure 2, left. Let $\psi(R)=R$ and, for $\rho \neq R$, let $\psi(\rho)$ be such that the straight line through $(\rho, q(\rho))$ and $(\psi(\rho), q \circ \psi(\rho))$ is tangent to the graph of $q$ at $(\psi(\rho), q \circ \psi(\rho))$. Besides, for $\bar{\rho} \in\left[0, R_{T}[\right.$ the line through $(\bar{\rho}, q(\bar{\rho}))$ and $(\psi(\bar{\rho}), q \circ \psi(\rho))$ has a further intersection with the graph of $q$, which we call $(\phi(\bar{\rho}), q \circ \phi(\bar{\rho}))$. Introduce two thresholds $s$ and $\Delta s$ such that

$$
s>0, \Delta s>0, s<R_{M} \text { and } R>s+\Delta s \geq \phi(s)>R_{T}>R-\Delta s .
$$



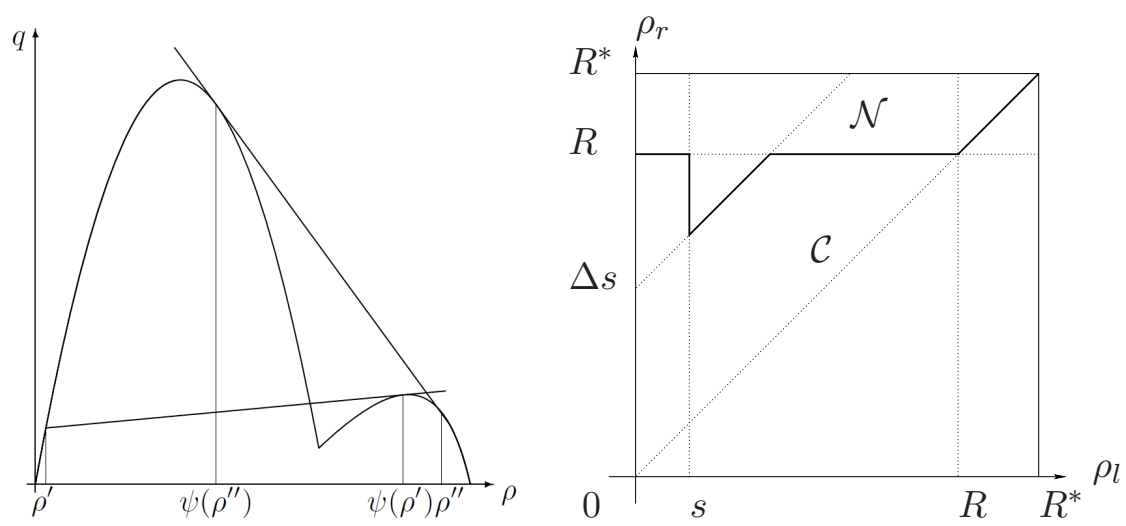

Fig. 2. Left, the function $\psi$ : its geometrical meaning. Right, the Riemann Solver: in $\mathcal{C}$, the solution consists of classical waves only; in $\mathcal{N}$, also nonclassical shocks are present.

We are now ready, following [11], to state the properties that allow to define $\mathcal{R}$, see [10] for a slightly more general presentation, though limited to the Riemann Problem.

(R.1) If $\rho^{l}, \rho^{r} \in[0, R]$, then $\mathcal{R}\left(\rho^{l}, \rho^{r}\right)$ selects the classical solution unless

$$
\rho^{l}>s \text { and } \rho^{r}-\rho^{l}>\Delta s .
$$

In this case, $\mathcal{R}\left(\rho^{l}, \rho^{r}\right)$ consists of a nonclassical shock between $\rho^{l}$ and $\psi\left(\rho^{l}\right)$, followed by the classical solution between $\psi\left(\rho^{l}\right)$ and $\rho^{r}$.

(R.2) If $\rho^{r}<\rho^{l}$, then $\mathcal{R}\left(\rho^{l}, \rho^{r}\right)$ is the classical solution.

(R.3) If $R \leq \rho^{l}<\rho^{r}$ or $\rho^{l}<R<\rho^{r}$ and the segment between $\left(\rho^{l}, q\left(\rho^{l}\right)\right)$ and $\left(\rho^{r}, q\left(\rho^{r}\right)\right)$ does not intersect $q=q(\rho)$, then the solution is a shock between $\rho^{l}$ and $\rho^{r}$.

(R.4) If $\rho^{l}<R<\rho^{r}$ and the segment between $\left(\rho^{l}, q\left(\rho^{l}\right)\right)$ and $\left(\rho^{r}, q\left(\rho^{r}\right)\right)$ intersects $q=q(\rho)$, then $\mathcal{R}\left(\rho^{l}, \rho^{r}\right)$ consists of a nonclassical shock between $\rho^{l}$ and a panic state followed by a possibly null classical wave. More precisely,

$\left.\rho^{r} \in\right] R, \psi\left(\rho^{l}\right)\left[: \mathcal{R}\left(\rho^{l}, \rho^{r}\right)\right.$ consists of a nonclassical shock between $\rho^{l}$ and $\psi\left(\rho^{l}\right)$, followed by a decreasing rarefaction between $\psi\left(\rho^{l}\right)$ and $\rho^{r}$;

$\rho^{r} \in\left[\psi\left(\rho^{l}\right), R^{*}\left[: \mathcal{R}\left(\rho^{l}, \rho^{r}\right)\right.\right.$ consists of a single nonclassical shock.

The Riemann Solver defined above is represented in Figure 2, right. According to [10, Theorem 2.1], there exists a unique Riemann Solver defined by (R.1)-(R.4) which is consistent and $\mathbf{L}_{\text {loc }}^{1}$ continuous in $\mathcal{\mathcal { C }}$ and $\mathcal{\mathcal { N }}$, see Figure 2, right. Recall that consistency is a necessary condition for the $\mathbf{L}^{\mathbf{1}}$ 
stability of the Cauchy Problem for (1.1). Refer to [10, I and II] or [8, Definition 2.2] for its precise statement.

In the next theorem, we refer to $[11,24]$ for the precise statement of the conditions on the flow function. Here, it is sufficient to recall that both diagrams in Figure 1 do satisfy to all of them.

Theorem 2.2. [11, Theorem 3.4] Let $f$ satisfy [10, (Q.1)-(Q.9)]. Let s, $\Delta s$ satisfy (2.6) and assume that there exists a constant $W$ satisfying

$$
W>1 \quad \text { and } \quad \frac{\phi(0)}{\psi(0)} \leq \frac{W+1}{2 W} \leq \frac{\Delta s}{\psi(s)-s} .
$$

Then, for any initial datum $\bar{\rho} \in\left(\mathbf{L}^{\mathbf{1}} \cap \mathbf{B V}\right)\left(\mathbb{R} ;\left[0, R^{*}\right]\right)$, the Cauchy Problem for (1.1) admits a nonclassical weak solution $\rho=\rho(t, x)$ generated by the nonclassical Riemann Solver $\mathcal{R}$ and defined for all $t \in \mathbb{R}^{+}$. Moreover:

$$
\operatorname{TV}(\rho(t)) \leq W \cdot \operatorname{TV}(\bar{\rho}), \quad \text { for all } t \in \mathbb{R}^{+} .
$$

\section{Passing through a Toll Gate}

This section is devoted to some numerical integrations of (2.3). Our aim is only to show that this model features reasonable qualitative properties, hence we choose normalized parameters, leaving to future works the quantitative agreement with experimental data.

Let the real interval $[0,2]$ describe a segment of a highway with a toll gate at its center $x=1$, as in Figure 3 The evolution of traffic is described

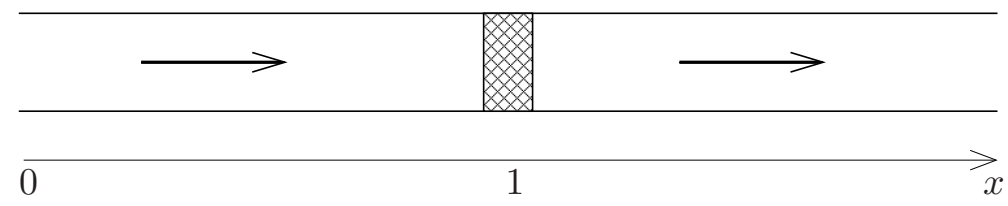

Fig. 3. A toll gate sited at $x=1$.

by (2.3) with, for instance, $f(\rho)=\rho(1-\rho)$. For simplicity, we assume that at time $t=0$ vehicles are uniformly distributed between $x=0.2$ and the site of the gate $x=1$.

Assume that the initial density distribution is $\rho_{o}(x)=0.3$ for $x \in[0.2,1]$ and $\rho_{o}(x)=0$ for $x \in[0,2] \backslash[0.2,1]$. The threshold of the through flow at the gate is 0.1 . Then, the computed time necessary for all the vehicles to pass the toll gate is $t \approx 2.4$ and the evolution described by (2.3) is displayed in Figure 4. As it has to be expected, the toll gate causes the rise of a queue to the left of the gate. This queue first increases an then decreases, finally disappearing when all vehicles passed the gate. 

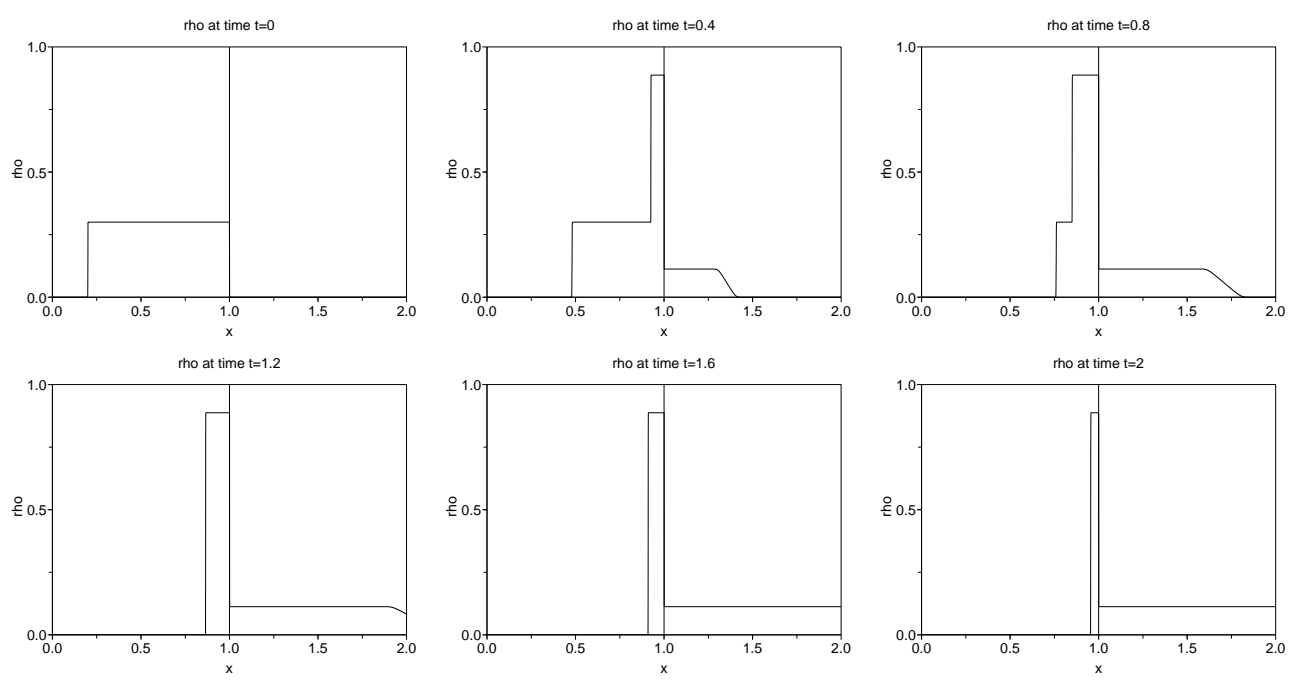

Fig. 4. Numerical integrations of (2.3) using Rusanov scheme, with $f(\rho)=\rho(1-\rho)$; $\rho_{o}(x)=0.3$ for $x \in[0.2,1], \rho_{o}(x)=0$ for $x \in \mathbb{R} \backslash[0.2,1]$ and $q(t)=0.1$. The constraint at $x=1$ is treated as suggested in [1].

We now let the initial density $\rho_{o}$ of vehicles and the efficiency of the gate $q$ vary, while keeping the other parameters fixed. The time $T$ that is necessary for all vehicles to pass the gate is then a function of $\rho_{o}$ and $q$, that is $T=T\left(\rho_{o}, q\right)$. As it has to be expected, this function is monotone in both variables, see Figure 5 . Note that as $q \rightarrow 0$, obviously, $T \rightarrow+\infty$. Hence, in
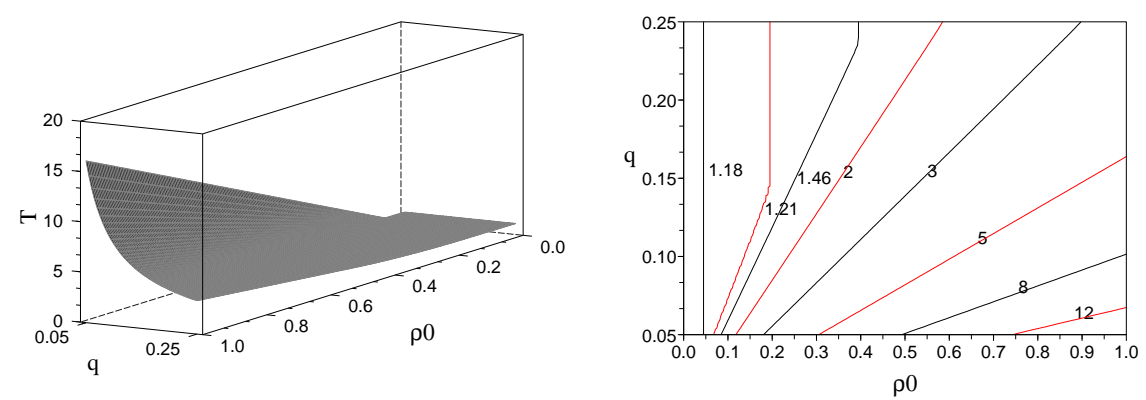

Fig. 5. A density of $\rho_{o} \in[0.1,1]$ vehicles is uniformly distributed on $[0.2,1]$. A toll gate is sited at $x=1$ and its through flow is $q \in[0.05,0.25]$. $T$ is the time necessary for all vehicles to pass the gate. Left, 3D diagram and, right, the level curves with $\rho_{o}$ on the horizontal axis and $q$ on the vertical one.

Figure 5, $T$ is computed only for $q \geq 0.04$. Note the vertical segments in the level curves of $T$ in Figure 5, right. They realistically correspond to the gate being sufficiently efficient to avoid the rising of queues. On the contrary, as 
soon as the toll gate influences the traffic flow, $T$ is well approximated by a function of the ratio $\rho_{o} / q$, as also dimensional considerations suggest.

\section{Pedestrian Evacuation and Braess Paradox}

Consider now a corridor with space coordinate, say, $x \in[0, L]$ with an exit at $x=D$, with $0<D<L$. Then, the dynamics of the crowd exiting the corridor is described by (2.3), with the standard Riemann Solver substituted by that prescribed through (R.1)-(R.4), see [11, Section 4.1] or [24, Section 2]. In emergency situations, it is well known that the pressure of the people seeking to exit may dramatically reduce the door efficiency. To prevent this, often suitable obstacles, typically columns, are posed in front of the exit, at a suitable distance, to partially sustain the crowd pressure.

In fact, the presence of an obstacle may avoid the insurgence of panic among the people, therefore keeping the door efficiency at a higher level. Paradoxically, the insertion of this obstacle may reduce the evacuation time, although most individuals may have a slightly longer path to reach the exit. This remarkable behavior reminds of the Braess paradox [2] typical of networks and is captured by the model presented in $[10,11,24]$.

Assume now that an obstacle is placed in the corridor above at, say, $x=d$, with $b<d<D$. A group of people is uniformly distributed on the

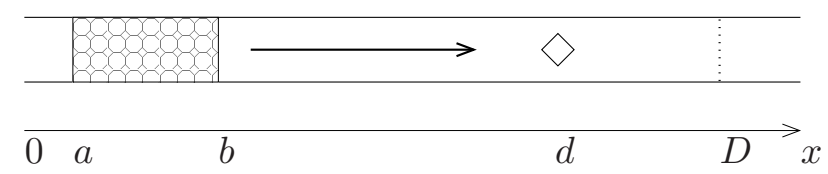

Fig. 6. A corridor with an obstacle before the exit.

segment $[a, b]$, with $0<a<b<d$, see Figure 6 . Following Section 2 above and [11, Section 4], one is thus lead to integrate

$$
\left\{\begin{array}{lr}
\partial_{t} \rho+\partial_{x} f(\rho)=0 & f(\rho(t, d-)) \leq q(\rho(t, d-)) \\
\rho(0, x)=\rho_{o}(x) & f(\rho(t, D-)) \leq Q(\rho(t, D-))
\end{array}\right.
$$

with the evolution prescribed by the Riemann Solver defined by (R.1)(R.4). The result is shown in Figure 7. As is well known, the efficiency of the exit may well be dramatically reduced when the crowd is panicking. Therefore, we assume that

$$
\begin{gathered}
q(\rho)=\left\{\begin{array}{lll}
\hat{q} & \text { if } & \rho \in[0, R] \\
\check{q} & \text { if } & \left.\rho \in] R, R^{*}\right]
\end{array} \quad \text { with } \quad \hat{q}>\check{q},\right. \\
Q(\rho)=\left\{\begin{array}{lll}
\hat{Q} & \text { if } & \rho \in[0, R] \\
\check{Q} & \text { if } & \left.\rho \in] R, R^{*}\right]
\end{array} \text { with } \quad \hat{Q}>\check{Q} .\right.
\end{gathered}
$$



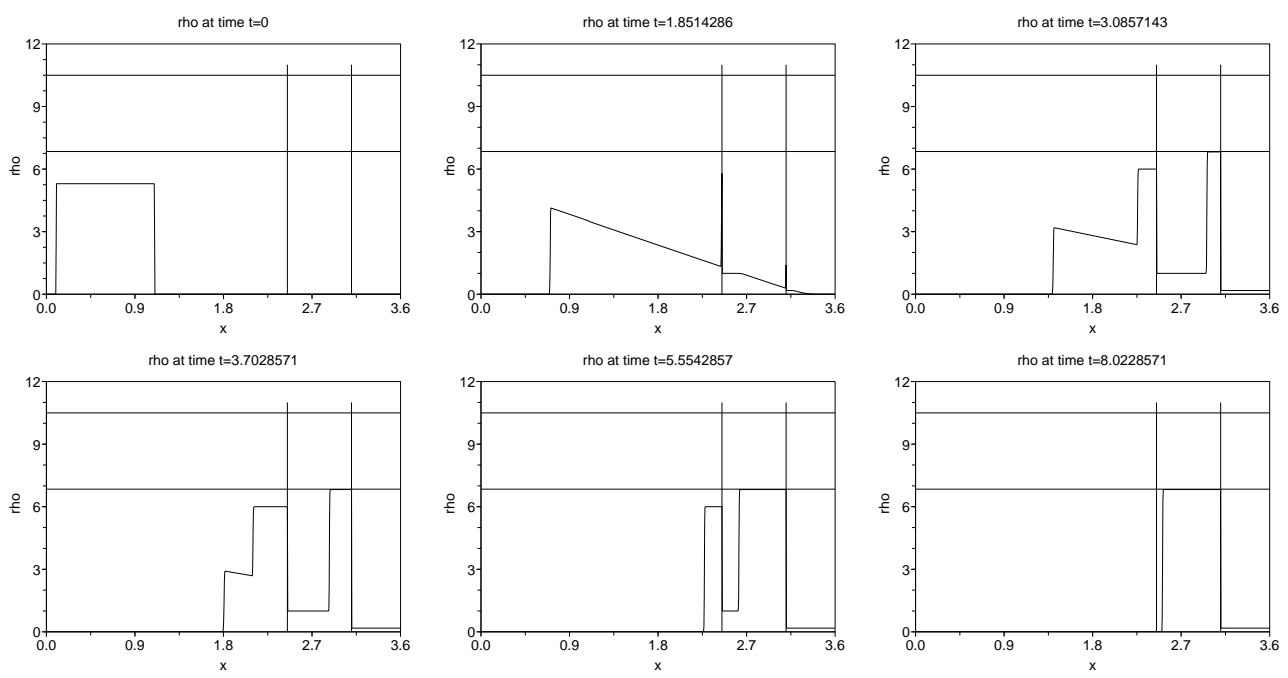

Fig. 7. Numerical integrations of (4.9) using the scheme in [4]. The vertical segments denote the positions of the obstacle and of the exit. The horizontal segments denote the maximal non-panic density $R$ and the overall maximal density $R^{*}$. Here: $a=0.1, b=1.1$, $d=2.45, D=3.1$ and $L=3.6$. The constraints at $x=d, D$ are treated as in [1].

The evacuation time $T$ is particularly relevant and can be computed integrating (4.9)-(4.10) numerically following the procedure used in Section 3. Having a simple initial datum, i.e. uniformly distributed on a given segment, an analytical study is also possible. Indeed, the wave front tracking technique applied to (4.9)-(4.10) yields Figure 8, right. In Figure 8, left, the same problem (4.9)-(4.10) is considered, but the obstacle is removed, i.e. $q>\max (\rho v(\rho))$. Remarkably, in this particular situation, the evacuation time with no obstacle is larger than the evacuation time with the obstacle. The detailed construction of these solutions can be found in [11, Section 4.2]. Note that the darker regions in Figure 8, left, represent where the crowd density attains panic values, i.e. $\left.\rho \in] R, R^{*}\right]$. The presence of the obstacle avoids the density to reach these high values, thus allowing for a faster evacuation from the room. In particular, we get the diagram in Figure 9 for the evacuation time, $T$, as a function of the position of the obstacle, $d \in] b, D[$. Note that when the obstacle is too near to the exit, i.e. to the right of the point $K$ in Figure 8, left, its presence becomes negligible. Indeed, at $K$ panic arises, while the efficiency of the obstacle is primarily dependent on avoiding it. 

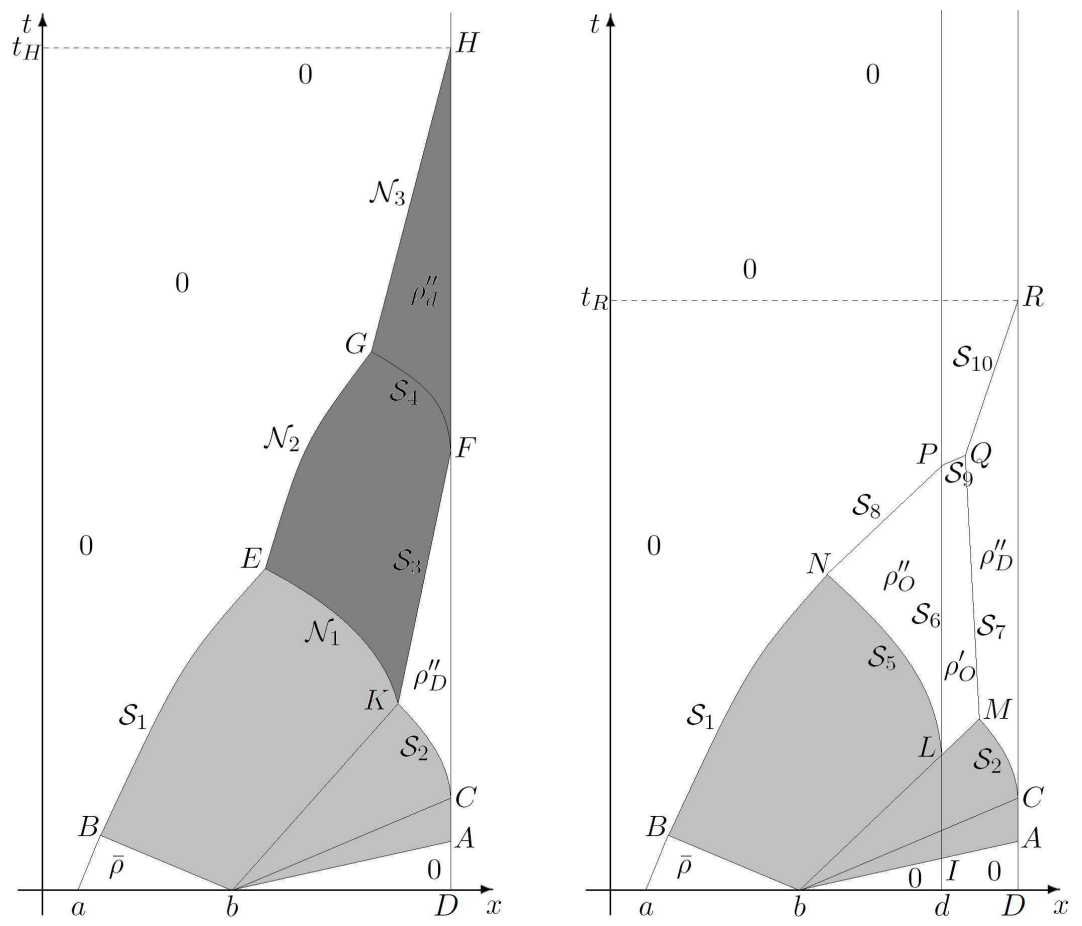

Fig. 8. Left, the structure of the solution to (4.9)-(4.10) with no obstacle, i.e. with $q>\max (\rho v(\rho))$; the evacuation time is $t_{H}$. Right, the case with the obstacle, the evacuation time is $t_{R}$.

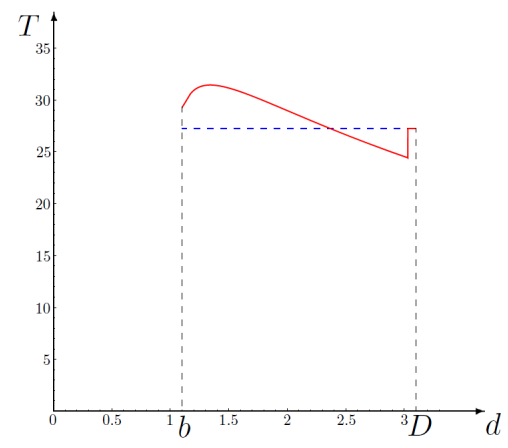

Fig. 9. The dotted line is the evacuation time with no obstacle. The solid line is the evacuation time, $T$, as a function of the position of the obstacle, $d$. When the obstacle is too near to the exit, its presence becomes negligible.

\section{Conclusions}

We presented some analytical results on unilateral constraints in scalar conservation laws. They can be used to model real situations, such as 
1. traffic flowing across a toll gate;

2. crowd evacuating a corridor.

In both cases, numerical integrations are possible, allowing a detailed description of the phenomena. Furthermore, the times necessary for vehicles to cross the gate or for the people to exit the room can be computed.

Reasonable qualitative behaviors of the solutions are described. In particular, in the case of pedestrians exiting a room, the model presented accounts for the possible decrease in the evacuation time thanks to the careful insertion of an obstacle at a well chosen position in front of the exit. This phenomenon, an analog of Braess paradox [2], is clearly non generic.

Acknowledgments. The second author was partially supported by I.N.R.I.A. Sophia Antipolis - Méditerranée.

\section{REFERENCES}

1. Boris Andreianov, Paola Goatin and Nicolas Seguin. Finite volume schemes for locally constrained conservation laws. In preparation.

2. Dietrich Braess. Über ein Paradoxon aus der Verkehrsplanung. Unternehmensforschung, 12:258-268, 1968.

3. Alberto Bressan, Hyperbolic systems of conservation laws. Oxford University Press, 2000.

4. Christophe Chalons. Numerical approximation of a macroscopic model of pedestrian flows. SIAM J. Sci. Comput., 29(2):539-555 (electronic), 2007.

5. Christophe Chalons and Paola Goatin. Godunov scheme and sampling technique for computing phase transitions in traffic flow modeling. Interfaces and Free Boundaries, 10(2):195-219, 2008.

6. Christophe Chalons and Paola Goatin. Transport-equilibrium schemes for computing contact discontinuities in traffic flow modeling. Commun. Math. Sci., 5(3):533-551, 2007.

7. Giuseppe M. Coclite and Nils H. Risebro. Conservation laws with time dependent discontinuous coefficients. SIAM J. Math. Anal., 36(4):12931309 (electronic), 2005.

8. Rinaldo M. Colombo and Paola Goatin. A well posed conservation law with a variable unilateral constraint. J. Differential Equations, 234(2):654-675, 2007.

9. Rinaldo M. Colombo and Graziano Guerra On General Balance Laws with Boundary. Preprint, arXiv:0810.5246v1, 2008.

10. Rinaldo M. Colombo and Massimiliano D. Rosini. Pedestrian flows and non-classical shocks. Math. Methods Appl. Sci., 28(13):1553-1567, 2005. 
11. Rinaldo M. Colombo and Massimiliano D. Rosini. Existence of nonclassical solutions in a pedestrian flow model. Nonlinear Analysis: Real World Applications, to appear.

12. Constantine M. Dafermos, Hyperbolic conservation laws in continuum physics. Springer-Verlag, 2000.

13. François Dubois and Philippe Lefloch. Boundary conditions for nonlinear hyperbolic systems of conservation laws. J. Differential Equations, 71(1):93-122, 1988.

14. Dirk Helbing. A fluid-dynamic model for the movement of pedestrians. Complex Systems, 6(5):391-415, 1992.

15. Dirk Helbing, Illès Farkas and Tamàs Vicsek. Simulating dynamical features of escape panic. Nature, 407, September 28th 2000.

16. Dirk Helbing, Anders Johansson and Habib Zein Al-Abideen. Dynamics of crowd disasters: An empirical study. Physical Review E, 75(2), 2007.

17. Serge Hoogendoorn and Piet H. L. Bovy. Pedestrian route-choice and activity scheduling theory and mode. Transp. Res. B, 2002.

18. Serge Hoogendoorn and Piet H. L. Bovy. Simulation of pedestrian flows by optimal control and differential games. Optimal Control Appl. Methods, 24(3):153-172, 2003.

19. Roger L. Hughes. A continuum theory for the flow of pedestrians. Transportation Research Part B, 36:507-535, 2002.

20. Stanislav N. Kružhkov. First order quasilinear equations with several independent variables. Mat. Sb. (N.S.), 81 (123):228-255, 1970.

21. Philippe G. Lefloch. Hyperbolic systems of conservation laws. Lectures in Mathematics ETH Zürich. Birkhäuser Verlag, Basel, 2002. The theory of classical and nonclassical shock waves.

22. Michael J. Lighthill and Gerald B. Whitham. On kinematic waves. II. A theory of traffic flow on long crowded roads. Proc. Roy. Soc. London. Ser. A., 229:317-345, 1955.

23. Paul I. Richards. Shock waves on the highway. Operations Res., 4:4251, 1956.

24. Massimiliano D. Rosini. Nonclassical interactions portrait in a pedestrian flow model. J. Differential Equations, 246:408-427, 2009.

25. Blake Temple. Global solution of the Cauchy problem for a class of $2 \times 2$ nonstrictly hyperbolic conservation laws. Adv. in Appl. Math., $3(3): 335-375,1982$. 